\title{
Method for Determining the Valid Travel Route of Railways Based on Generalised Cost under the Syncretic Railway Network
}

\author{
Zhiqiang Tian $\mathbb{D}^{1},{ }^{1}$ Guofeng Sun $\mathbb{D}^{1},{ }^{1}$ Dingjun Chen $\mathbb{D}^{2},{ }^{2}$ Zhicheng Qiu, ${ }^{1}$ and Yawen $\mathrm{Ma}^{3}$ \\ ${ }^{1}$ School of Traffic and Transportation, Lanzhou Jiaotong University, Lanzhou 730070, China \\ ${ }^{2}$ School of Transportation and Logistics, Southwest Jiaotong University, Chengdu 610031, China \\ ${ }^{3}$ School of Traffic and Transportation, Beijing Jiaotong University, Beijing 100044, China \\ Correspondence should be addressed to Zhiqiang Tian; tianzq@mail.lzjtu.cn
}

Received 19 July 2019; Revised 18 November 2019; Accepted 29 November 2019; Published 11 January 2020

Academic Editor: Luca D’Acierno

Copyright (c) 2020 Zhiqiang Tian et al. This is an open access article distributed under the Creative Commons Attribution License, which permits unrestricted use, distribution, and reproduction in any medium, provided the original work is properly cited.

Travel route options for passengers can provide data support for railway line planning, passenger flow organisation, and train operation establishment. A critical review of the literature indicates that previous studies mainly focused on choices offered by a single railway network path without much consideration of China's normal-speed and high-speed integrated railway network and the effect of train timetable on passengers' travel choice. In this study, a method based on generalised cost is proposed to discover the valid routes of passenger travel in the integrated network of China's normal-speed and high-speed railways. After quantifying the effects of train fare, travel time, transfer, travel convenience, comfort, and other factors on the generalised expenses of passengers, this study presents a generalised cost determination method when individuals select an option from different seats of different trains of specific railway transport products. Theoretically, the valid routes considering the train schedule is defined, and a valid route search algorithm is designed using the deep traversal idea in a new valid route searching network. Considering the Lanzhou-Beijing passenger travel routes as an example, this study verifies the practicability of the generalised cost calculation method, as well as that of the valid routes search method.

\section{Introduction}

High-speed railway, as a land transportation mode with high speed, large capacity, low energy consumption, and environmental protection, is setting off a wave of development all over the world. As the country with the longest operating mileage of normal-speed and high-speed railways worldwide, China's normal-speed railway and high-speed railway operation mileages reached 132,000 and 30,000 kilometres, respectively, by the end of 2018 [1]. Therefore, railway networks formed by high-speed and normal-speed railways have begun to expand. Under this network condition, ways of making the passenger decision-making service more demand-responsive have stood under the spotlight. Therefore, as a base of the railway passenger decision-making service, it is essential to study the railway passenger path choice.
Scholars have studied passenger travel route selection, including the physical route selection of passengers and passenger trains. Based on the behaviour of passenger path selection, $\mathrm{Xu}$ et al. [2] proposed a method of random delay path selection of trains based on reliability and established a model of travel choice of road network passengers, considering heterogeneous passengers. van Loon et al. [3] studied the effect of travel time reliability on railway passenger travel and established six travel time reliability indexes for analysing the preference of passenger travel route selection. Lim et al. [4] analysed a postdisaster evacuation route selection using three binary logit route selection models. Two models were found to be useful in providing relevant suggestions on evacuation time, route, and destination for postdisaster route selection. Moghaddam et al. [5] investigated a travel purpose based on a simulated driver experiment. The travel time reliability and income were 
found to be important factors that affect the route selection of road traffic passengers.

For passengers who choose rail as their primary means of transport, the physical path only determines whether the passengers can get to their respective destination stations from the departure station. However, in the railway network, a physical route usually runs multiple trains with varying grades, speeds, and costs. Consequently, the travel path based on the physical network cannot reflect the transfer behaviour of long-distance passengers. Several scholars have studied the route selection of passengers in the case of train schedules. Shi et al. [6] proposed a traffic-type assignment algorithm based on route choice by defining the transfer reliability using passengers' expected reliability threshold to choose the lowest cost path. When calculating the passenger travel expenses, they found that the generalised cost function mainly depends on the cost of travel time, ticket prices, and discomfort. Dou et al. [7] built a railway passenger travel network based on a train timetable, taking the generalised cost function and remaining train capacity as the basis of passenger travel path search. They proposed an optimal path search algorithm for railway passenger transport network based on the residual train capacity. By analysing the main factors that affect the route choice behaviour of passengers, Yang et al. [8] established an optimisation model for optimising the route selection for railway passengers by minimising the total travel time, total price cost, transfer time, transfer distance, and fatigue recovery time.

Some studies of passenger travel paths also considered the train timetable. For example, by using the smart card data of an urban rail transit, Wu et al. [9] proposed a method considering the uncertainty of walking time and transfer time simultaneously and estimated the travel time of all possible travel paths from the origin to destination. More accurate studies of passenger path matching and passenger flow distribution have been carried out. Similarly, based on passenger travel data, Zhang et al. [10] proposed a set of novel methods for predicting the passenger travel path selection in urban rail transits. Furthermore, $\mathrm{Xu}$ et al. [11] investigated the route selection behaviour of urban rail transit passengers using ticket price, train schedule, and train passenger flow data by combining Bayesian reasoning and urban congestion sampling.

When the train timetable is used for selecting passengers' travel routes, travel cost is usually the most critical factor in choosing a route, followed by ticket cost and time cost. Earnhart [12] chose channel cost, transportation cost, and time cost as the components of generalised cost in a constructed generalised cost model, and by detailed analyses, he concluded that time cost is the most crucial factor. De Borger and Fosgerau [13] divided the conversion methods of travel time and cost into four categories: willingness to pay (WTP), willingness to accept compensation (WTA), equivalent gain (EG), and equivalent loss (EL). Other researchers have further studied time cost. Deserpa [14] refined the concept of time cost and defined it in three kinds, namely, time cost as a resource, as a commodity, and time spent on an activity being less than a given time. Hess et al. [15] applied the hybrid logit algorithm to estimate the cost of saved travel time using the strategy of WTP and provided an estimation model with income and travel distance as independent variables for calculating the cost of saved travel time. Although many scholars have studied passenger travel behaviours, only a few studies considered the transfer, comfort, convenience, and other costs in the passenger travel process.

When considering travel costs in route selection, it is theoretically possible to use the exhaustive method of searching all the paths in the network to select the best path. However, the massive travel paths generated in either the railway network or urban traffic network will reduce the operating efficiency of this method. Therefore, many scholars proposed search algorithms for different paths in networks [16, 17]. Modesti and Sciomachen [18] proposed a multicriterion shortest path search method for urban traffic networks to minimise the total path cost. Samà et al. Sama et al. [19] studied the real-time train route choice problem in the railway system, where the core problem was converted into an integer linear programming problem and solved using the ant colony algorithm.

Most researchers have studied the passenger travel routes generation of normal-speed railway network and high-speed railway network separately, whereas the influence of the integration of the two-railway network is rarely taken into account. However, in the syncretic railway network, the possibilities for creating transfer between different lines have expanded, which makes it more difficult to calculate the generalised cost of passenger travel route. In this study, when searching the valid routes based on the train timetable, the adoption of ellipse method and angle method may increase the number of invalid nodes and missing valid partial routes. This study integrates the high-speed and normal-speed railway networks and then presents a detailed study of all the components (including fare, time, convenience, and comfort) of the generalised cost of efficient travel routes. Finally, considering the current train timetable, the generalised cost is used to design a searching algorithm for the effective transfer routes search based on the nodes of each direct route. A deep traversal search technique is applied to determine all the reasonable passenger travel routes.

The significant contributions of this study are as follows: (1) the calculation method of the generalised cost is proposed, including the calculation methods of comfort cost, convenience cost, and transfer cost, and (2) A method of passenger travel routes search between the Origin-Destination (OD) considering the train schedule under the dualnetwork integration is proposed.

This paper is organised as follows. The calculation method of the generalised cost of railway passenger travel is discussed in Section 2. The valid routes search based on the generalised cost is analysed and discussed in Section 3, considering the actual train timetable. Section 4 presents a summary of the results and recommends suggestions for future research.

\section{Generalised Cost Function}

In this study, the generalised cost of each OD travel includes the following five parts: fare, time, convenience, transfer, and comfort values. 
2.1. Fare. The passengers travelling fares between OD stations consists of three parts, namely, the travel cost from the origin to departure station, railway specific product cost, and cost taken from the terminal railway station to the destination, as presented in Figure 1 and equation (1):

$$
C_{\text {cost }}=C_{O}+C_{R}+C_{D}
$$

where $C_{\text {cost }}$ is the total fare during the trip; $C_{R}$ is the fare of a particular train service; $C_{O}$ is the fare from origin $O$ to departure railway station; $C_{D}$ is the fare from the terminal railway station to the destination $D$.

2.2. Travel Time Cost. Professionally, the passenger's travel time cost is calculated by the production method, which is defined as the cost that can be created if the time expended by the passenger during the journey is spent on productive activities. The travel time that determines the corresponding cost [20] varies from passenger to passenger. The calculation formula of travel time value per unit is listed as follows:

$$
V(T)=\frac{\text { GDP }}{(T \times P)},
$$

where GDP refers to the gross national product and $T$ and $P$ are the average working hours and population, respectively.

The total travel time for passengers is the sum of the travel time from the origin to departure station, from the departure to the terminal station (including transfer), and from the terminal station to the destination:

$$
C_{\text {time }}=\left(T_{O S}+T_{S_{s} S_{e}}+T_{S D}\right) V(T) \text {, }
$$

where $C_{\text {time }}$ is the time cost of passengers; $V(T)$ is the travel time cost parameter; $T_{O S}$ is the travel time from the origin to the departure station; $T_{S_{s} S_{e}}$ is travel time between the tworailway stations; and $T_{S D}$ is the travel time from the terminal station to the destination.

2.3. Convenience Cost. The travel convenience cost of passengers mainly deals with the issue of whether the departure and arrival times match the service time of the urban public transport system (bus or subway). If the departure or arrival time is within the operational range of the urban public transport, the time is considered to be relatively convenient, and the corresponding convenience cost is comparatively lower. Otherwise, the time will be considered inconvenient and increases the convenience cost.

The urban public transport operation time considering the remarkable volatility of passenger flow can be divided into peak hours and off-peak hours. Specifically, it is quite convenient to travel with a little cost during the nonpeak periods. However, the convenience cost increases rapidly during rush hours because of the massive passenger flow and traffic congestion.

To sum up, the convenience cost can be categorised as hours with no public transport service, peak hours, and offpeak hours, whose respective costs reduce gradually. For urban residents, set the convenience cost during off-peak hours as $\phi$ assuming the service period of the bus is equal to

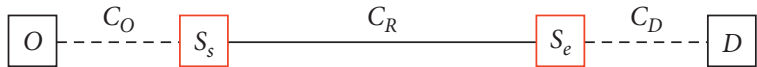

\begin{tabular}{|l|l}
\hline$O$ & Origin \\
\hline$D$ & Destination \\
\hline$S_{s / e}$ & Railway station
\end{tabular}

FIGURE 1: Structural sketch of passenger fare composition.

that of the urban rail transit. Consequently, the convenience cost of the passenger departure time $C_{\text {convience }}^{\text {dep }}$ for each period can be designed as follows:

$C_{\text {convience }}^{\text {dep }}= \begin{cases}\phi, & \text { passengers depart during off - peak hours, } \\ \delta_{1} \phi, & \text { passengers depart during peak operating hours, } \\ \delta_{2} \phi, & \text { passengers depart during nonoperating hours, }\end{cases}$

where $\delta_{1}$ and $\delta_{2}$ are the convenience cost coefficients of peak hours and the hours when no public transport is available, respectively.

During the travel, the convenience value of arrival $C_{\text {convience }}^{\text {arr }}$ is designed similar to that of departure. Thus, the total convenience value $C_{\text {convenience }}$ can be calculated as follows:

$$
C_{\text {convenience }}=C_{\text {convenience }}^{\mathrm{dep}}+C_{\text {convenience }}^{\mathrm{arr}}
$$

2.4. Transfer Cost. During the travelling process, the costs of the railway transfer value have three parts, namely, the transfer cost from the urban transportation to railway products, the internal transfer cost in the railway terminals, and the transfer cost from the railway products to urban traffic, as illustrated in Figure 2. The total transfer cost $C_{\text {transfer }}$ from the origin to destination can be formulated as follows:

$$
C_{\text {transfer }}=C_{\text {transfer }}^{O S_{s}}+C_{\text {transfer }}^{S_{t}}+C_{\text {transfer }}^{S_{e} D}
$$

where $C_{\text {transfer }}^{O S_{s}}$ is the cost of the transfer value from the urban traffic to railway departure stations; $C_{\text {trangffer }}^{S_{t}}$ is the transfer cost generated in the railway network; $C_{\text {transfer }}^{e_{S}}$ is the transfer cost from railway arrival stations to urban traffic. The three indicators can be quantitatively calculated as follows.

2.5. Calculation of $C_{\text {transfer }}^{O S_{s}}$ and $C_{\text {transfer. }}^{S_{e} D}$. For passengers travelling by train, the transfer value between the urban traffic and railway service is principally determined by the transfer time. Hence, the value is the product of the transfer time and time value coefficient.

(1) Transfer cost from urban traffic to railway $C_{\text {transfer }}^{O S_{s}}$ is

$$
C_{\text {transfer }}^{O S_{s}}=V(T) T_{\text {transfer }}^{O S_{s}} .
$$

Here, $T_{\text {transfer }}^{O S_{s}}$ is the total transfer time from the urban traffic to the railway station, which is mainly composed 


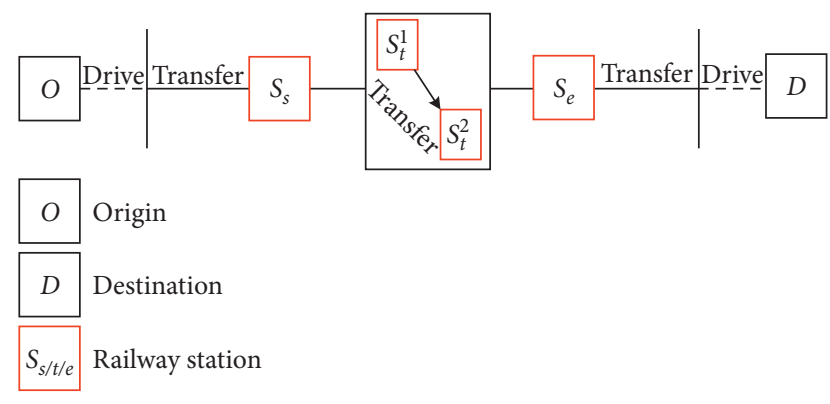

Figure 2: Passenger transfer cost diagram.

of the transfer travel time and the waiting time as follows:

$$
T_{\text {transfer }}^{O S_{s}}=T_{\text {walking }}^{O S_{s}}+T_{\text {waiting }}^{O S_{s}}
$$

Here, $T_{\text {walking }}^{\mathrm{OS}_{\mathrm{s}}}$ is the walking time from the urban traffic platform to the railway station (or the waiting room at the railway station). $T_{\text {waiting }}^{O S_{s}}$ is the average waiting time for connecting two different transportation systems.

(2) Similarly, the transfer cost from the railway system to the urban traffic $C_{\text {transfer }}^{S_{S} D}$ can be designed as follows:

$$
\begin{aligned}
& C_{\text {transfer }}^{S_{e} D}=V(T) T_{\text {transfer }}^{S_{e} D}, \\
& T_{\text {transfer }}^{S_{e} D}=T_{\text {walking }}^{S_{e} D}+T_{\text {waiting }}^{S_{e} D}
\end{aligned}
$$

where $T_{\text {transfer }}^{S_{e} D}$ is the total transfer time from the railway network to the urban traffic; $T_{\text {walking }}^{S_{S} D}$ is the walking time from the railway station platform to the urban traffic; and $T_{\text {waiting }}^{S_{e} D}$ is the waiting time connecting the transfer.

2.6. Calculation of $C_{\text {transfer }}^{S_{t}}$. An extended period may be taken to transfer from one train to another at the railway station due to transfer failure. This case is different from the transfer between the urban traffic and railway transportation. Therefore, the accommodation and transportation costs $C_{\text {transfer }}^{\text {night }}$ in the city where the transfer station is located should be considered.

$$
C_{\text {transfer }}^{S_{t}}=C_{\text {transfer }}^{\text {night }}+V(T) T_{\text {transfer }}^{S_{t}}
$$

Here, $C_{\text {transfer }}^{S_{t}}$ is the cost generated in the entire transfer process at the railway terminal (transfer taking place at the different stations in a city) or railway station (transfer taking place at the same station), $T_{\text {transfer }}^{S_{t}}$ is the transfer time between the arriving and departing trains, and $C_{\text {transfer }}^{\text {night }}$ is the accommodation cost in the city where the transfer node is located. The equation for calculating $C_{\text {transfer }}^{\text {night }}$ is expressed as follows:

$$
C_{\text {transfer }}^{\text {night }}= \begin{cases}\Psi, & \text { transfer is not successful on this day, } \\ 0, & \text { transfer is successful on this day, }\end{cases}
$$

where $\Psi$ is the cost of the accommodation and transportation supposing that the transfer is unsuccessful on the day in the node city.

The transfer time from the arrival train to the departure train is composed of the transfer walking time and transfer waiting time, that is,

$$
T_{\text {transfer }}^{S_{t}}=T_{\text {walking }}^{S_{t}}+T_{\text {waiting }}^{S_{t}}
$$

where $T_{\text {walking }}^{S_{t}}$ is the travel time at the transfer station (city) and $T_{\text {waiting }}^{S_{t}}$ is the waiting time for the following train.

2.7. Comfort Cost. Along the journey, the comfort level depends typically on factors such as the train class, seat type, travel time, and travel conditions, among which the seat type and travel time are the top prioritised factors. For passengers travelling during the day at a short travel time, the comfort level of the first-class seat (ZY) and secondclass seat (ZE) of the running train-set on the high-speed lines is usually more preferable to that of hard seat and hard sleeper. When high-speed railways undergo maintenance at night during 0:00-6:00 hours (the skylight period), travellers can only choose the normal-railway train services with only the hard seat (YZ), hard sleeper (YW), and soft berth (RW) being available. When considering the comfort value cost during travels, a more comfortable journey generates lower comfort cost. The comfort level order is RW, YW, and YZ. In this study, assuming the highest comfort score is 10 and defining the comfort value coefficient as constant $\mathrm{A}$, the passenger comfort value $C_{\text {comfortable }}$ can be determined by the following function:

$$
C_{\text {comfortable }}=A\left(10-X_{i}^{t}\right),
$$

where $X_{i}^{t}$ is the comfort value of the seat type $i$ corresponding to the travel time. For the first-class seats, secondclass seats, and hard seats, the comfort level is relatively acceptable within a specified time range. However, the comfort level will decline sharply when the travel time increases and exceeds a particular critical value. For hard and soft sleepers, the comfort level varies linearly with an increase in travel time.

Depending on whether the passengers can have a good rest during the journey, the seat types can be roughly divided into two categories. The seats, including ZY, ZE, and YZ, belong to one category, whereas the other category includes YW and RW. The passenger comfort value of the different seat types during various travel times can be obtained after processing the data from a questionnaire survey, as listed in Table 1.

In this study, the efficiency and convenience are directly measured by the price simultaneously. The time value cost, transfer value cost, and comfort value cost are quantified by the time cost together with the normalisation by time value coefficient. A unified dimension can be adopted for these five generalised costs. Therefore, the total generalised costs can be defined as follows: 
TABLe 1: Costs of comfort level.

\begin{tabular}{|c|c|c|c|c|c|c|c|}
\hline \multirow{2}{*}{\multicolumn{2}{|c|}{ Comfort }} & \multicolumn{6}{|c|}{ Travel time (h) } \\
\hline & & {$[0-2]$} & {$[2-4]$} & {$[4-6]$} & {$[6-8]$} & {$[8-10]$} & $>10$ \\
\hline \multirow{3}{*}{ High-speed railway } & First seat $(\mathrm{ZY})$ & 9.8 & 9.0 & 8.3 & 7.2 & 6.1 & 4.9 \\
\hline & Second seat (ZE) & 9.1 & 8.2 & 6.9 & 5.8 & 4.9 & 3.8 \\
\hline & Hard seat (YZ) & 6.9 & 6.1 & 5.1 & 4.2 & 3.1 & 1.8 \\
\hline \multirow[t]{2}{*}{ Common railway } & Hard sleeper (YW) & 9.2 & 8.5 & 8.2 & 7.5 & 6.9 & 6.5 \\
\hline & Soft sleeper (RW) & 9.9 & 9.5 & 8.9 & 8.5 & 8.0 & 7.5 \\
\hline
\end{tabular}

$$
C=C_{\text {cost }}+C_{\text {time }}+C_{\text {convenient }}+C_{\text {transfer }}+C_{\text {comfortable }} \text {. }
$$

For example, an individual travels through the LanzhouBeijing section. Assuming that the fare and travel cost from origin ( $O$, home or office) to the railway station equals that of the railway station to destination ( $D$, home or office), the fare is $¥ 20$, and the travel time is $0.75 \mathrm{~h}$. If the other parameters are set as follows: $\phi=¥ 3, \quad \delta_{1}=1.5, \quad \delta_{2}=10$, $T_{\text {walking }}^{O S}+T_{\text {waiting }}^{O S}=1 \mathrm{~h}, \quad A=50, \quad \mathrm{GDP}=90.0309$ trillion, $T=8 \mathrm{~h}, P=1.390$ billion, then the generalised cost of all seat types on different trains in the syncretic railway network can be determined, as presented in Table 2.

As can be seen in Table 2, the generalised cost components of seat types of the normal-speed railway vary with those of the high-speed railway. The price of the normalspeed railway is much lower than that of the high-speed railway, and its time value cost is much higher than that of high-speed railway. In terms of comfort value cost, the transfer between normal-speed railway and high-speed railway is more friendly than that of the normal-speed railway or high-speed railway only. During the transferring process, the generalised cost is usually higher than that of the route travel.

\section{Valid Route Search in the Syncretic Railway Network}

3.1. Valid Route in the Syncretic Railway Network. The valid routes concept has been theoretically studied and obtained mature works [21], deriving from traffic allocation issues. Under the double-network integration condition, the valid route is defined as follows. When travellers choose a railway travel product, the train number and different seats constitute the travel routes. Meanwhile, it is assumed that the route impedance $e$ is within the $(1+H)$ period of shortest routes, namely, the valid route threshold $C \leq C_{\min }(1+H)$, where $H$ is a nonnegative constant representing the route stretching coefficient. To determine the valid routes between specific ODs in the railway network, it is first required to find the shortest routes between the ODs, as well as all other possible routes. If the possible routes meet certain conditions, in practice, they can be regarded as valid routes.

In the valid route definition above, the value of the extension coefficient $H$ directly determines the number of valid routes. When $H=0$, only a few routes based on the shortest routes can be found. The smaller the value of $H$, the smaller the number of valid routes. With an increase in $H$, the number of valid routes increases. In this study, the value of $H$ influences the operation mileage. The values of coefficient $H$ for different travel mileages are listed in Table 3.

3.2. Construction of Route Searching Network. When passengers choose railway products for travelling, they rely on operational railway lines and trains. Under the fixed conditions of line facilities, the train operation charts directly guide the selection of passenger travel routes. When passengers select a railway from $\mathrm{O}-\mathrm{D}$, they can choose a through route or transfer route. The route should be the shortest route based on generalised cost. When they choose the through route, there are two options: the through route of normal-speed railway or high-speed railway. If the transfer route is selected, there are three options: the transfer between normal-speed railway lines, between high-speed railways lines, or between both high-speed railway lines and normal-speed railway lines. The railway network for selecting the OD passenger travel route is depicted in Figure 3. From the perspectives of $S_{S^{-}} S_{e}$ and physical routes, passengers can choose multiple routes, such as $S_{S}-1-2-3-S_{e}$, $S_{S^{-}}-4-7-S_{e}$, and $S_{S^{-}} 5-4-7-S_{e}$. However, from the perspective of the railway service department, a farther physical route increases the travel ticket price, time cost, and generalised cost. Consequently, few passengers will likely select these unreasonable routes.

In the physical travel route, assuming that $S_{S^{-}}-1-2-3-S_{e}$ is the shortest physical route and this route runs through normal-speed and high-speed railways, passengers can choose the normal-speed railway route $S_{S^{-}} 1-2-3-S_{e}$ or highspeed railway route $S_{S}-1-2-3-S_{e}$. When considering the timetable of normal-speed and high-speed railways, in addition to the through route, the $\mathrm{O}-\mathrm{D}$ route selection scheme will become more complex. Passengers can follow the normal-speed train from $S_{s}$ to city node 1 and then transfer to another normal-speed train or high-speed train to $S_{e}$. Passengers can choose the high-speed railway train from $S_{s}$ to city node 2 and then transfer to the subsequent high-speed train or the subsequent normal-speed train to arrive at $S_{e}$. The partial train timetable considering the syncretic railway network between $S_{s}-S_{e}$ is displayed in Figure 4 .

Based on a train timetable, this study investigated the passenger travel route. On the physical network formed by the syncretic normal-speed and high-speed railways, considering that there will be multiple railway stations at different city nodes under the syncretic networks, it will expand the space and establish the time-space service network of passenger travel route. For passengers travelling through the physical network integrated by the normal-speed and high- 
TABLe 2: Generalised cost calculation table (unit: $¥$ ).

\begin{tabular}{lccccccccc}
\hline Train number & \multicolumn{2}{c}{ K886 } & \multicolumn{2}{c}{ G428 } & \multicolumn{2}{c}{ Z56 } & \multicolumn{3}{c}{ K680+ G658 } \\
Need transfer & \multicolumn{2}{c}{ No } & \multicolumn{2}{c}{ No } & \multicolumn{2}{c}{ No } & \multicolumn{3}{c}{ Yes } \\
Seat type & YZ & YW & ZY & ZE & YW & YZ + ZY & YZ + ZE & YW + ZY & YW + ZE \\
\hline$C_{\text {cost }}$ & 246 & 393 & 1096.5 & 730 & 362.5 & 957.5 & 648.5 & 1027.5 \\
$C_{\text {time }}$ & 822.23 & 822.23 & 286.16 & 286.16 & 527.81 & 527.81 & 504.98 & 504.98 & 504.98 \\
$C_{\text {convenience }}$ & 4.5 & 4.5 & 4.5 & 4.5 & 3 & 34.5 & 34.5 & 34.5 & 34.5 \\
$C_{\text {transfer }}$ & 57.06 & 57.06 & 57.06 & 57.06 & 57.06 & 275.31 & 275.31 & 275.31 & 275.31 \\
$C_{\text {comfortable }}$ & 400 & 175 & 200 & 250 & 175 & 550 & 600 & 350 & 400 \\
$C$ & 1529.79 & 1451.79 & 1644.22 & 1327.72 & 1125.37 & 2322.29 & 2063.29 & 2192.29 & 1933.29 \\
\hline
\end{tabular}

TABLE 3: Variation table of route extension coefficient (unit: km).

\begin{tabular}{lcccccc}
\hline OD distance $(\mathrm{km})$ & $0-200$ & $200-400$ & $400-700$ & $700-1000$ & $1000-1500$ & $>1500$ \\
\hline$H$ & 1 & 0.92 & 0.85 & 0.7 & 0.5 \\
\hline
\end{tabular}

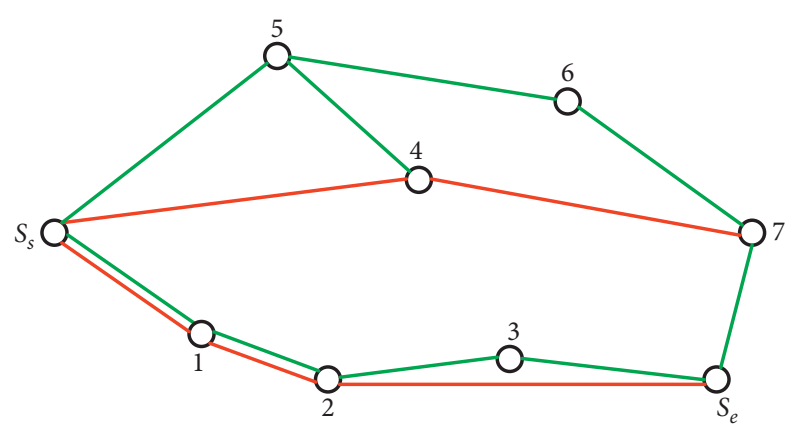

— Normal-speed railway line

— High-speed railway line

FIgURE 3: Simple road network of a railway under the syncretic network.

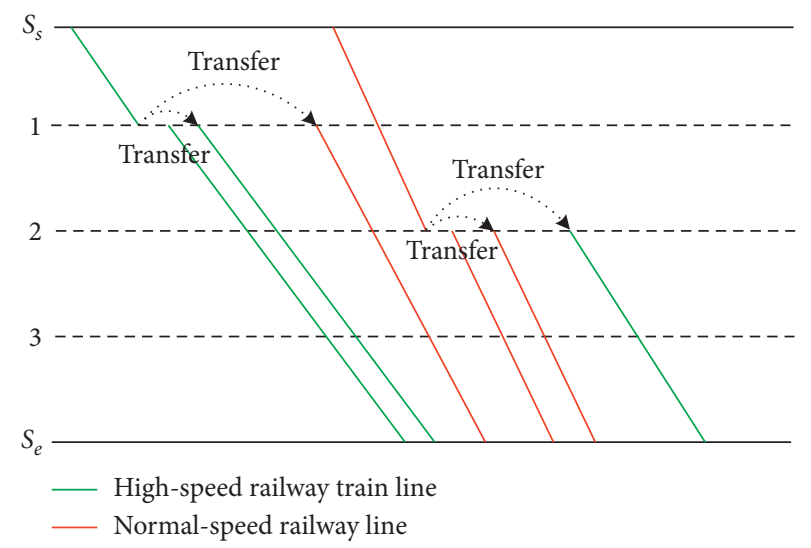

FIgURE 4: Simplified train timetable considering transfer.

speed railway lines, assuming that the route choices between OD pairs, including the normal-speed and high-speed railways are built, the physical network can be constructed, as shown in Figure 5(a). The physical routes from the origin station (with normal-speed railway and high-speed railway) to the destination station (also with normal-speed railway and high-speed railway) can be represented as $S_{S^{-}} 1-2 / 2^{\prime}-3 /$ $3^{\prime}-S_{e}$. It can be noticed that the number of passenger travel routes based on the physical network is quite small.
On the contrary, for the integrated double-network mentioned above, the passenger travel constructed network based on the train timetable is large-scale and complex. In addition to the direct travel schemes, the transfer plans between the high-speed trains, normal-speed trains, or both become available, as illustrated in Figure 5(b).

In this study, it is assumed that only one transfer is possible for the passengers in the course of the journey. All the routes between $S_{s}$ and $S_{e}$ in the syncretic railway network, including the direct routes and transfer routes, are shown in Figure 6. Figure 6 also presents the routing network based on the train timetable of each $S_{s}-S_{e}$; accordingly, all the routes can be found between OD in the syncretic railway network.

The parameters of the network for the railway passenger travel service are defined as follows:

$G=(N, A)$ : the passenger travel service network and simultaneously a directed graph composed of the train service nodes $N$ and train service arcs $A$ between the nodes.

$N$ : the set of all nodes in the travel service network.

$A$ : the set of all service routes in the travel service network, $A=\left\{a_{1}, a_{2}, \ldots\right\}$.

$a$ : the set of serving arc, $a=\left(s_{s}, s_{e}, t\right)$, where $s_{s}$ is the head node of arc $e, s_{e}$ is the tail node, and $t$ represents the arc of the train routes $t$.

$C\left(s_{s}, s_{e}, t\right)$ : the set of generalised cost of train $t$ serving arc $a$ between the start node $s_{s}$ and end node $s_{e}$.

$R_{s_{s} s_{e}}$ : the set of all valid routes from node $s_{s}$ to $s_{e}$.

3.3. Valid Route Search Algorithm. In the syncretic railway network, the transfer routing search results in difficulty for searching the valid routes based on generalised cost, and the number of transfer schemes is primarily affected by the transfer time and number of stations. A higher number of transfer stations passing through the travel routes results in a more complicated transfer route search. Generally, the success of the transfer depends on the transfer connection time, which should be set within a realistic range. Otherwise, 

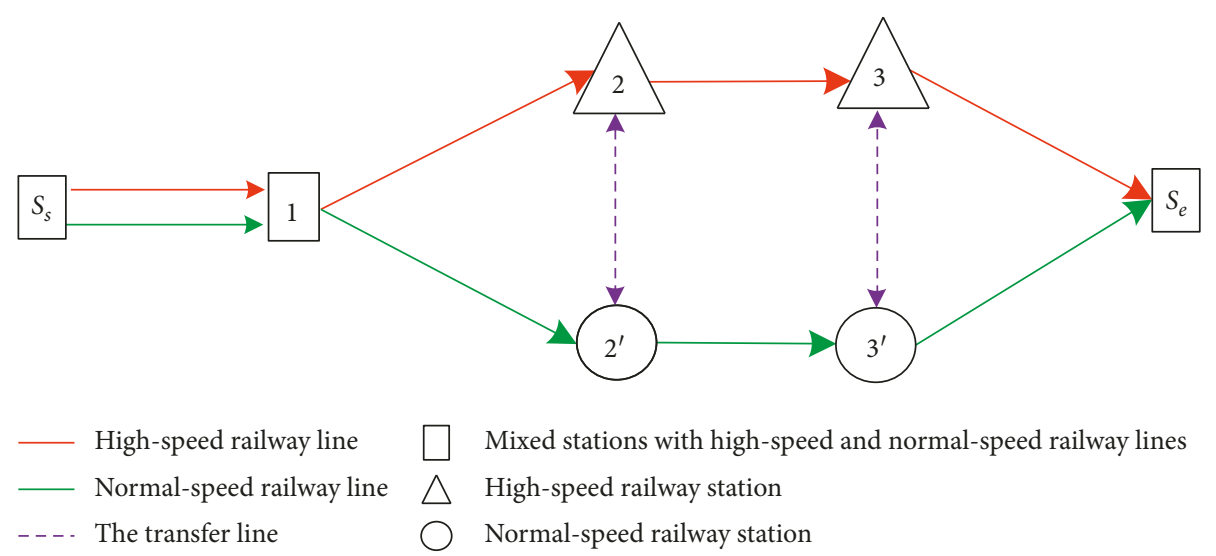

(a)

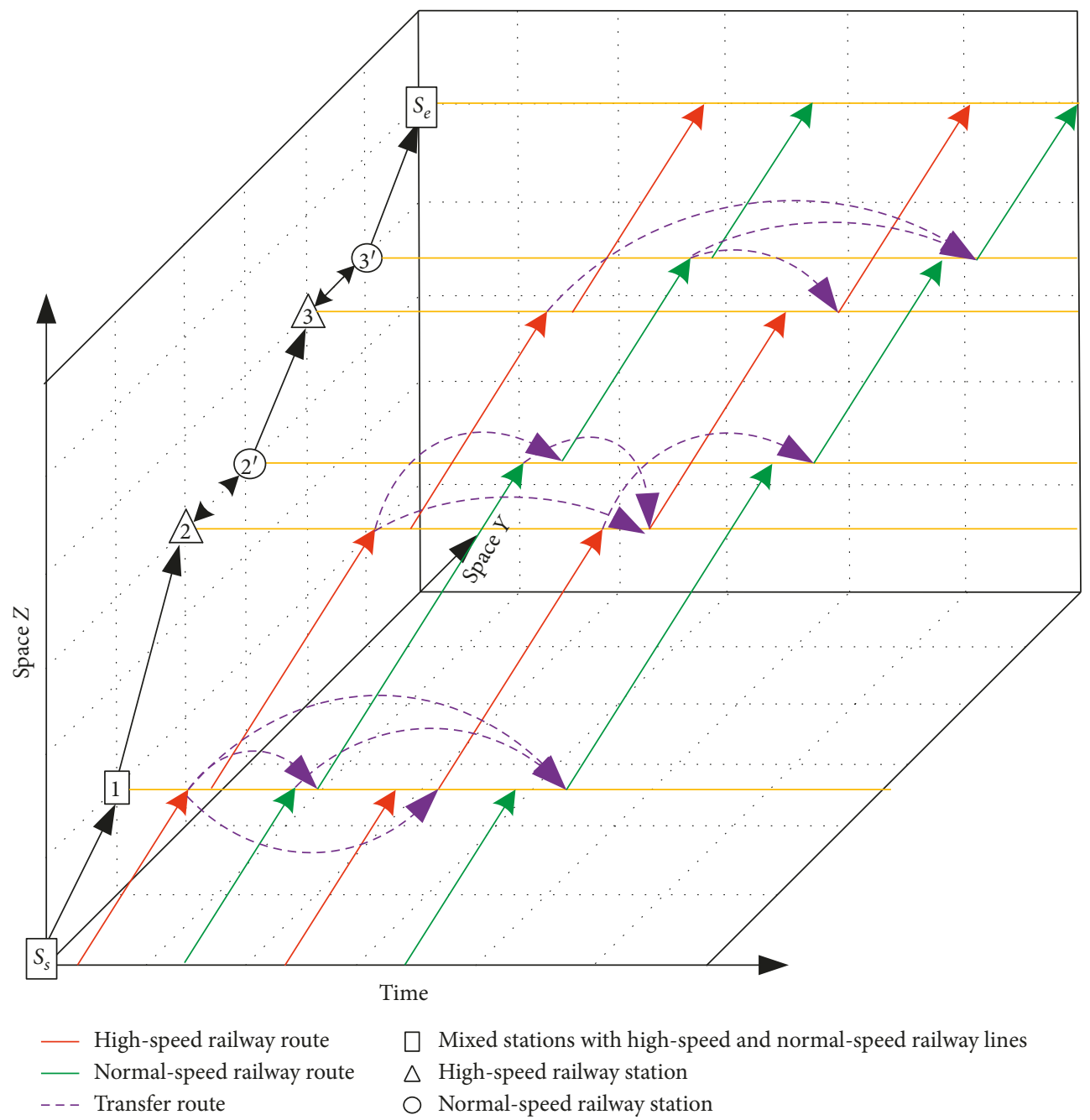

(b)

FIgURE 5: Complex train routes network diagram considering passengers' travel choice in the context of syncretic railway network. (a) Physical network structure diagram between nodes. (b) A complex train routing network diagram for train timetable.

the number of valid routes will increase sharply when the transfer connection time is prolonged.

Practically, all valid routes are searched by the depth-first traversal algorithm. The idea behind this algorithm is as follows. First, examine all the valid routes using the ergodic method and determine whether the routes meet the discriminant criterion. If yes, the routes are recorded and put in the set of valid routes; otherwise, the searching process is repeated in the previous layer of nodes. The method is applied repeatedly until all valid routes are discovered. 


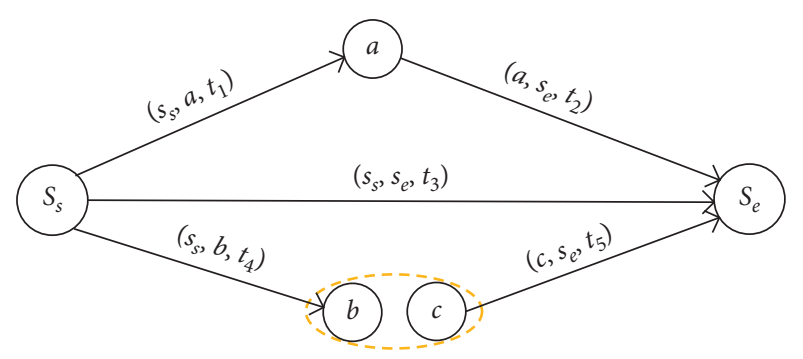

Figure 6: Passenger travel routes diagram.

In previous studies on the valid route of railway passenger travel, if the network fusion is neglected, the number of trains running on the existing line or high-speed railway line is limited. To obtain the feasible route within the shortest route $(1+H)$ time of the distance, researchers obtained more $k$ short routes using angle search or ellipse search techniques. However, for dual network convergence, the passenger train service (train number) between a specific OD is usually distributed on the shortest route. To obtain a lower ticket price and shorter travel time (two significant factors in the generalised cost), passengers do not usually choose the route outside the existing train service between specific OD. Although the threshold determination method of $(1+H)$ is also used in this study, this method is used for searching the feasible transfer route (or trip train number chain) based on the specific operation line, rather than when searching the physical route. In detail, the algorithm is designed as follows:

Step 1 (initialisation): initialise the value of the extension coefficient $H$ of the valid routes. Set the transfer time $\tau_{h}$ between the maximum and minimum limit reasonably, that is, $\tau_{\min } \leq \tau_{h} \leq \tau_{\max }$.

Step 2: search all the through routes. After calculating the generalised cost of all the through routes between specific OD pairs based on the train timetable, set the generalised cost of the first route as the minimum cost. Replace the minimum route with a smaller route after a repeated calculation during the loop (if any). Check and update the strategy and obtain the minimum generalised cost for the normal-speed railway $C_{\min }^{1}$ and highspeed railway $C_{\min }^{2}$. Put all direct routes into set $E$ and select all the valid routes to set $R_{s_{s} s_{e}}$, according to the judgement criterion.

Step 3: search all the transfer routes. In the syncretic railway network, there are two kinds of transfer, namely, the internal transfer within the normal-speed railway network, that of the high-speed railway network, and the external transfer between the normalspeed and high-speed railway networks. Therefore, find all the transfer routes of four specific transfer patterns: the route from normal-speed railway to normal-speed railway, or high-speed railway, or the route from highspeed railway to normal-speed railway, or high-speed railway. For all of these transfers, the judgement criteria for valid routes are the constraint of transfer time.

Step 3.1: search the internal transfer routes. According to the train timetable, if the number of transfers and the transfer time meet the specified standard ranges, then all the transfer routes of the node where the routes stop between the OD will be searched. It will then be analysed whether the routes can be regarded as valid routes. Repeatedly, all transferable nodes where the through routes pass will be obtained and added to set $A$ at the end.

Step 3.2: search the external transfer routes. Unlike internal transfer, the external transfer requires further consideration of transfer plan occurring at different stations within the same city. Based on the train timetable, search the common stations (unique type of stations where both normal-speed and high-speed trains stop, which can also be called mixed stations) or the cities where both the normal-speed through train and high-speed through train pass. Then, locate all possible transfer connections in the mixed station (or each transfer city) restricted by the transfer time and put all found routes in set $A$.

Step 3.3: analyse the valid routes in set $A$. For a given $H$, the internal transfer route validity in the normalspeed railway network by $C_{\min }^{1}$ and high-speed railway network, respectively, will be analysed. In terms of the external transfer routes in set $A, C_{\min }^{1}+C_{\min }^{2} / 2$ will be taken as the judging criteria to determine whether the routes are valid. Subsequently, all the valid routes can be put in set $R_{s_{s} s_{e}}$.

Step 4: obtain all the feasible route set $A$ and valid route set $R_{s_{s} s_{e}}$ for the particular OD pair.

\section{Computational Experiments}

To verify the accuracy of the proposed valid route search algorithm, the syncretic railway network from Lanzhou to Beijing was determined in this study, using the train timetable of March 2019. Figure 7 illustrates a simplified Lanzhou-Beijing railway network for both normal-speed and high-speed railway lines.

From the search results, 7,218 travel routes were available for passengers from Lanzhou to Beijing, among which 2,195 were valid routes, including 18 direct routes and 2,177 transfer routes. For a simpler representation, the head and tail nodes of the valid routes are omitted, and the nodes are represented by train numbers. The travel through routes (corresponding to the direct train services) of both normal-speed and high-speed railways from Lanzhou to Beijing are shown in Table 4.

From Table 4, the minimum generalised cost of the through route on normal-speed railway obtained by the searching algorithm is $C_{\min }^{1}=E 1175.24$ (less than $¥ 1645.34$, the criteria for valid route determination). Thus, it can be identified as a valid route. Different from the actual timetable, neither of through train K886 and K4238 is a valid route. The reason is that both trains take a roundabout route, which depart from Lanzhou, stop at Yinchuan, Baotou, Huhehaote, and Datong during the journey and finally arrive at Beijing. Therefore, their travel time cost (28 hours) is much larger, compared with that of through train Z22 and Z56 (16.5 hours). 


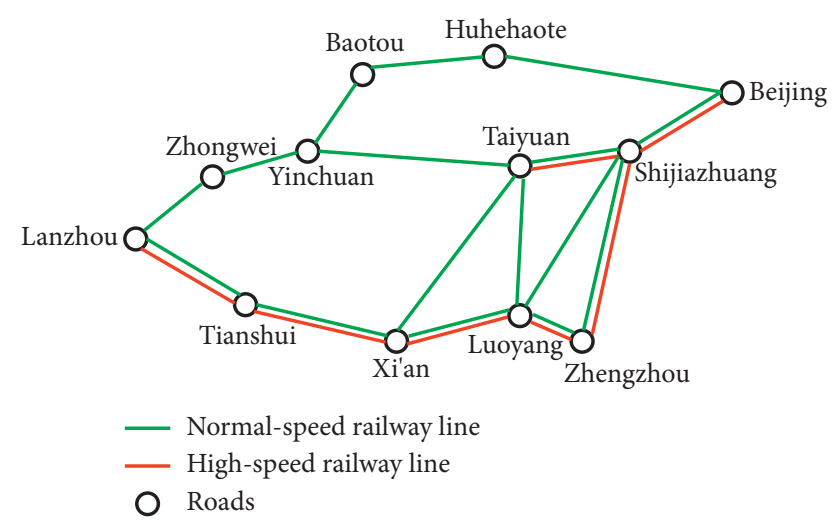

Figure 7: Simplified Lanzhou-Beijing road network map under syncretic railway network.

TABLe 4: Direct routes search results.

\begin{tabular}{lccc}
\hline Number & Routes & Generalised cost & Whether effective \\
\hline 1 & T176 (YZ) & 1615.37 & 1 \\
2 & T176 (YW) & 1408.87 & 1 \\
3 & T176 (RW) & 1541.87 & 1 \\
4 & K886 (YZ) & 2443.58 & 0 \\
5 & K886 (YW) & 1658.58 & 0 \\
6 & K886 (RW) & 1767.58 & 0 \\
7 & K4238 (YZ) & 2050.07 & 0 \\
8 & K4238 (YW) & 1762.57 & 0 \\
9 & K4238 (RW) & 1922.07 & 0 \\
10 & Z22 (YZ) & 1306.74 & 1 \\
11 & Z22 (YW) & 1175.24 & 1 \\
12 & Z22 (RW) & 1308.24 & 1 \\
13 & Z152 (YZ) & 1629.38 & 1 \\
14 & Z152 (YW) & 1441.88 & 1 \\
15 & Z152 (RW) & 1601.38 & 1 \\
16 & Z76 (YZ) & 1623.11 & 1 \\
17 & Z76 (YW) & 1435.61 & 1 \\
18 & Z76 (RW) & 1595.11 & 1 \\
19 & Z56 (YW) & 1214.51 & 1 \\
20 & Z56 (RW) & 1347.51 & 1 \\
21 & G428 (ZY) & 1686.30 & 1 \\
22 & G428 (ZE) & 1369.80 & 1 \\
21 & G430 (ZY) & 1675.46 & 1 \\
22 & G428 (ZE) & 1358.96 & 1 \\
\hline
\end{tabular}

To further verify the accuracy of the method, a questionnaire survey was carried out, and the results indicate that among all the passengers, $98.6 \%$ prefer not to choose train K886 and K4238. When the tickets of the other through trains (with shorter travel time if possible) on normal-speed railway lines are sold out, $57.5 \%$ prefer to stop and transfer at Xi'an, Zhengzhou, and other city nodes, whereas $41.1 \%$ prefer to follow high-speed trains. A survey of the passengers taking trains K886 and K4238 reveals that the destinations of the trains departing from Lanzhou are mainly Yinchuan, Baotou, or Huhehaote, and the destination of the trains departing from Baotou and Huhehaote is usually Beijing. Therefore, K886 and K4238 trains are not in the valid route set from Lanzhou to Beijing. As for the high-speed railway lines from Lanzhou to Beijing, the minimal generalised cost of a through train service $C_{\min }^{2}=E 1358.96$. The through travel routes for any seat type on train G428 and G430 are valid routes.

With a traversal of all the transfer stations (cities), a total of 2,183 transfer routes were found. Some of the routes are listed in Table 5.

By analysing the valid transfer route from Lanzhou to Beijing, it is clear that none of the transfer routes passing through Baotou or Huhehaote are in the valid route set. Moreover, the travel costs are high compared with those of other through trains and transfers at other nodes. The reason for this high cost is because the transfer cost at Baotou station where the Baolan and Jingbao rail lines intersect is relatively high owing to the long travel distances, prolonged travel time, and absence of high-speed railway available for transfer. In other words, as both K886 and K4238 through train services are invalid routes, transfer routes along K886 and $\mathrm{K} 4238$ are invalid.

An analysis of the search results reveals that under dualnetwork integration conditions, no valid transfer routes exist from normal-speed railway to high-speed railway at the following city (station) nodes: Tianshui, Xi'an, and Luoyang. The major reason is that the generalised cost from Lanzhou to the city (station) nodes mentioned above by a normalspeed railway (Longhai line) is much higher than that by high-speed railway. Taking Xi'an as an example, it takes 8 hours to travel from Lanzhou to Xi' an along the Longhai line (normal-speed railway line) having a distance of $676 \mathrm{~km}$, and 3 hours from Lanzhou West Station to Xi'an North Station along Xulan high-speed railway line having a distance of $568 \mathrm{~km}$. The generalised travel cost from Lanzhou to Xi'an by normal-speed railway is much higher than that by highspeed railway due to the effect of travel time value and comfort on passengers.

The number of valid transfer routes at each transfer node obtained using the searching algorithm is shown in Table 6 .

From Table 6, Xi'an, Zhengzhou, and Shijiazhuang have the most valid transfer routes, whereas Yinchuan has the least valid routes. From the perspective of China's railway network structure, first, the three cities (stations) connect more railway lines (both by normal-speed railway and highspeed railway) and have several passenger trains passing through the stations. Second, the Xi'an node has almost all transfer options for individuals travelling from Lanzhou to Beijing; this also applies to the Zhengzhou and Shijiazhuang nodes. Third, a passenger can select a preferable transfer plan from several options at the three city (station) nodes with a low generalised cost when no through train service is available from the timetable.

In terms of the valid routes shown in Table 7 (from various means of transport), the number of valid routes by normal-speed railway is 14 , and its minimum generalised travel cost is $¥ 1175.24$. Similarly, the number of valid routes by high-speed railway is 4 , and its minimum generalised travel cost is $¥ 1358.96$. There are 262 and 965 valid internal transfer routes by normal-speed railway and high-speed railway, respectively. The number of valid mixed transfer routes between normal-speed railway and high-speed railway is 950 . 
TABle 5: Part transfer valid routes.

\begin{tabular}{|c|c|c|c|}
\hline $\mathrm{S} / \mathrm{N}$ & Valid routes & Transfer station & Generalised cost $(¥)$ \\
\hline 1 & K9882 (YW) + T176 (YW), Zhongwei & Zhongwei & 1510.41 \\
\hline 2 & K9882 (YW) + T176 (RW), Zhongwei & Zhongwei & 1637.47 \\
\hline 3 & K9882 (RW) + T176 (YW), Zhongwei & Zhongwei & 1531.47 \\
\hline 4 & Z56 (YW) + Z70 (YW), Zhongwei & Zhongwei & 1594.60 \\
\hline 5 & Z56 (RW) + Z70 (YW), Zhongwei & Zhongwei & 1591.60 \\
\hline 6 & K886 (YZ) + Z275 (YW), Yinchuan & Yinchuan & 1618.85 \\
\hline 7 & K886 (YW) + Z275 (YZ), Yinchuan & Yinchuan & 1583.35 \\
\hline 8 & K886 (YW) + Z275 (YW), Yinchuan & Yinchuan & 1476.35 \\
\hline 9 & K886 (YW) + Z275 (RW), Yinchuan & Yinchuan & 1574.35 \\
\hline 10 & K886 (RW) + Z275 (YZ), Yinchuan & Yinchuan & 1604.85 \\
\hline 11 & K886 (RW) + Z275 (YW), Yinchuan & Yinchuan & 1497.85 \\
\hline 12 & K886 (RW) + Z275 (RW), Yinchuan & Yinchuan & 1595.85 \\
\hline 13 & G866 (ZY) + G428 (ZE), Tianshui & Tianshui & 1592.29 \\
\hline 14 & G866 (ZE) + G428 (ZE), Tianshui & Tianshui & 1558.29 \\
\hline 15 & D8970 (ZE) + G430 (ZE), Tianshui & Tianshui & 1451.76 \\
\hline 16 & D2680 (ZE) + G428 (ZE), Tianshui & Tianshui & 1548.31 \\
\hline 17 & D2566 (ZY) + Z76 (YW), Tianshui & Tianshui & 1742.20 \\
\hline 18 & D2566 (ZE) + Z76 (YW), Tianshui & Tianshui & 1741.63 \\
\hline 19 & G834 (ZE) + Z152 (YW), Tianshui & Tianshui & 1754.56 \\
\hline 20 & D2680 (ZY) + G652 (ZE), Xi’an & Xi'an & 1529.42 \\
\hline 21 & D2680 (ZE) + G652 (ZE), Xi'an & Xi'an & 1522.42 \\
\hline 22 & D2654 (ZY) + G652 (ZY), Xi'an & Xi'an & 1744.29 \\
\hline 23 & D2654 (ZY) + G652 (ZE), Xi'an & Xi'an & 1485.29 \\
\hline 24 & $\mathrm{D} 2654(\mathrm{ZE})+\mathrm{G} 652(\mathrm{ZY}), \mathrm{Xi}$ an & Xi'an & 1737.29 \\
\hline 25 & D2654 (ZE) + G652 (ZE), Xi'an & Xi'an & 1478.29 \\
\hline 26 & D2556 (ZY) + Z20 (YZ), Xi'an & Xi'an & 1614.72 \\
\hline 27 & D2556 (ZY) + Z20 (YW), Xi'an & Xi'an & 1483.29 \\
\hline 28 & $\mathrm{D} 2556(\mathrm{ZE})+\mathrm{Z} 20(\mathrm{YW}), \mathrm{Xi}$ an & Xi'an & 1062.72 \\
\hline 29 & G866 (ZY) + G662 (ZY), Luoyang & Luoyang & 1762.45 \\
\hline 30 & G866 (ZY) + G662 (ZE), Luoyang & Luoyang & 1636.35 \\
\hline 31 & G866 (ZE) + G662 (ZY), Luoyang & Luoyang & 1755.08 \\
\hline 32 & G866 (ZY) + G662 (ZE), Luoyang & Luoyang & 1695.58 \\
\hline 33 & G866 (ZE) + G660 (ZE), Zhengzhou & Zhengzhou & 1580.84 \\
\hline 34 & G866 (ZE) + G660 (ZY), Zhengzhou & Zhengzhou & 1751.84 \\
\hline 35 & Z106 (YW) + Z36 (YZ), Zhengzhou & Zhengzhou & 1554.64 \\
\hline 36 & Z106 (YW) + Z36 (YW), Zhengzhou & Zhengzhou & 1480.20 \\
\hline 37 & G1840 (ZE) + G516 (ZY), Zhengzhou & Zhengzhou & 1601.07 \\
\hline 38 & G1840 (ZE) + G516 (ZE), Zhengzhou & Zhengzhou & 1465.17 \\
\hline 39 & Z106 (YW) + G588 (ZE), Zhengzhou & Zhengzhou & 1591.15 \\
\hline 40 & Z106 (YW) + G588 (ZY), Zhengzhou & Zhengzhou & 1727.15 \\
\hline 41 & D2566 (ZY) + G626 (ZY), Taiyuan & Taiyuan & 1630.18 \\
\hline 42 & D2566 (ZY) + G626 (ZE), Taiyuan & Taiyuan & 1561.68 \\
\hline 43 & D2566 (ZE) + G626 (ZY), Taiyuan & Taiyuan & 1515.68 \\
\hline 44 & D2566 (ZE) + G626 (ZE), Taiyuan & Taiyuan & 1447.18 \\
\hline 45 & $\mathrm{Z} 56(\mathrm{YW})+\mathrm{Z} 70(\mathrm{YZ})$, Taiyuan & Taiyuan & 1638.09 \\
\hline 46 & Z56 (YW) + Z70 (YW), Taiyuan & Taiyuan & 1550.59 \\
\hline 47 & $\mathrm{Z} 56(\mathrm{YW})+\mathrm{Z} 70(\mathrm{RW})$, Taiyuan & Taiyuan & 1572.59 \\
\hline 48 & Z56 (RW) + Z70 (YW), Taiyuan & Taiyuan & 1629.59 \\
\hline 49 & $\mathrm{Z} 22(\mathrm{YW})+\mathrm{G} 606(\mathrm{ZY})$, Taiyuan & Taiyuan & 1634.28 \\
\hline 50 & Z22 (YW) + G606 (ZE), Taiyuan & Taiyuan & 1565.78 \\
\hline 51 & G1840 (ZE) + G622 (ZY), Shijiazhuang & Shijiazhuang & 1578.50 \\
\hline 52 & G1840 (ZE) + G622 (ZE), Shijiazhuang & Shijiazhuang & 1510.50 \\
\hline 53 & Z22 (YW) + G8954 (ZY), Shijiazhuang & Shijiazhuang & 1538.68 \\
\hline 54 & Z22 (YW) + G8954 (ZE), Shijiazhuang & Shijiazhuang & 1510.68 \\
\hline 55 & G2096 (ZE) + K22 (YW), Shijiazhuang & Shijiazhuang & 1515.22 \\
\hline 56 & G2096 (ZE) + K22 (RW), Shijiazhuang & Shijiazhuang & 1468.22 \\
\hline
\end{tabular}

It should be noted that there are a few through travel routes from Lanzhou to Beijing, as well as a few valid transfer routes by normal-speed railway. The travel cost (especially that of transfer route) increases rapidly because of the low speed and long travel time. On one hand, due to the initially networked high-speed railway, there are several 
TABLE 6: Valid routes number for transfer nodes.

\begin{tabular}{lcccccccc}
\hline Transfer nodes & Zhongwei & Yinchuan & Tianshui & Xi'an & Luoyang & Zhengzhou & Taiyuan & Shijiazhuang \\
\hline Number of valid routes & 41 & 9 & 105 & 644 & 140 & 524 & 78 & 636 \\
\hline
\end{tabular}

TABLE 7: Number of valid routes from different means.

\begin{tabular}{|c|c|c|c|}
\hline \multicolumn{2}{|c|}{ Means of travel } & Number of valid routes & Minimum generalised costs $(¥)$ \\
\hline \multirow{2}{*}{ Normal-speed railway } & Through & 14 & \multirow{2}{*}{1175.24} \\
\hline & Transfer & 262 & \\
\hline \multirow{2}{*}{ High-speed railway } & Through & 4 & \multirow{2}{*}{1358.96} \\
\hline & Transfer & 950 & \\
\hline \multicolumn{2}{|c|}{$\begin{array}{l}\text { Mixed transfer between normal- and high-speed } \\
\text { railways }\end{array}$} & 965 & 1267.10 \\
\hline
\end{tabular}

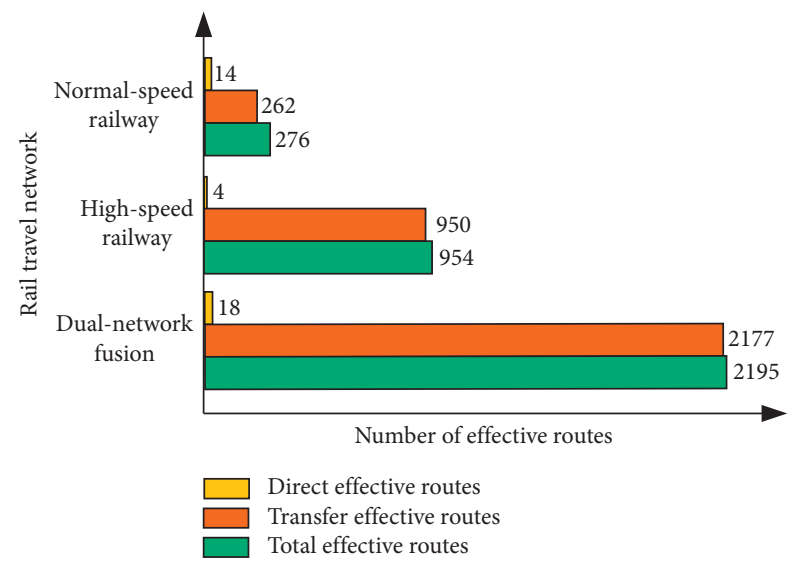

FIGURE 8: Comparison of the number of valid routes between single network and syncretic railway network.

transfer schemes among the high-speed railways. On the other hand, passengers tend to take transfer routes in case that through trains are unavailable owing to the mixed transfer plans at the nodes (such as Xi'an, Zhengzhou, and Shijiazhuang) where the normal-speed and high-speed railways intersect.

Figure 8 compares the number of valid routes among normal-speed railway network, high-speed railway network, and syncretic railway network. Considering only normalspeed or high-speed railway, the valid routes from Lanzhou to Beijing reached 1230 , including 276 produced by the normal-speed railway and 954 by high-speed railway. In the syncretic railway network, there were 2195 valid routes, which widens the travel plan choices of passengers and significantly improves travel convenience.

\section{Conclusions}

Through the analysis of each component of a generalised cost, such as fare, time, convenience, and transfer, this study presented a method to calculate the generalised cost in a syncretic railway network. Based on the minimum generalised cost calculated for different conditions and the preset cost of $H$, the valid routes with or without transfer from Lanzhou to Beijing were determined. The results show that in the syncretic railway network, the transfer plan between the normal-speed railway and high-speed railway significantly increases the number of valid routes.

Our study demonstrates that by integration of China's normal-speed and high-speed railway networks, better synergy should be considered in preparing the train timetabling process of the two networks.

In addition, the findings of this study can be applied to many aspects as follows:

(1) The calculated generalised cost can provide a favourable basis for passenger flow distribution and ticket allocation for the syncretic railway network.

(2) The calculated generalised cost can be used to evaluate the transportation efficiency of the railway network and provide a reference for railway network planning.

(3) According to the number of searched valid routes, the accessibility evaluation of railway network cities (stations) can be carried out.

(4) The conclusion of this study does not only provide a theoretical reference for China's railway operation but can also be extended to other countries with high-speed railway.

\section{Data Availability}

All data included in this study are available from the corresponding author upon request.

\section{Conflicts of Interest}

The authors declare that there are no conflicts of interest regarding the publication of this article.

\section{Acknowledgments}

This study was jointly supported by grants from the National Natural Science Foundation of China (grant no. 71761023) and Natural Science Foundation of Gansu Province, China (grant no. 18JR3RA110).

\section{References}

[1] Gov.cn, "Significant improvement of international position and sustainable enhancement of international influences: 
report 23 of the economic and social development achievement series for the 70th anniversary of the founding of new China," 2019, http://www.gov.cn/xinwen/2019-08/30/ content_5425839.htm.

[2] G. Xu, W. Liu, and H. Yang, "A reliability-based assignment method for railway networks with heterogeneous passengers," Transportation Research Part C: Emerging Technologies, vol. 93, pp. 501-524, 2018.

[3] R. van Loon, P. Rietveld, and M. Brons, "Travel-time reliability impacts on railway passenger demand: a revealed preference analysis," Journal of Transport Geography, vol. 19, no. 4, pp. 917-925, 2011.

[4] H. R. Lim, M. B. B. Lim, and M. Piantanakulchai, "Modeling route choice behavior of evacuees in highly urbanized area: a case study of Bagong Silangan, Quezon City, Philippines," Asia Pacific Management Review, vol. 24, no. 2, pp. 98-105, 2019.

[5] Z. R. Moghaddam, M. Jeihani, S. Peeta, and S. Banerjee, "Comprehending the roles of traveler perception of travel time reliability on route choice behavior," Travel Behaviour and Society, vol. 16, pp. 13-22, 2019.

[6] F. Shi, Z. Zhou, J. Yao, and H. Huang, "Incorporating transfer reliability into equilibrium analysis of railway passenger flow," European Journal of Operational Research, vol. 220, no. 2, pp. 378-385, 2019.

[7] F. Dou, K. Yan, Y. Huang, L. Wang, and L. Jia, "Optimal path choice in railway passenger travel network based on residual train capacity," The Scientific World Journal, vol. 2014, Article ID 153949, 8 pages, 2014.

[8] X. Yang, L. Liu, Y. Li, and R. He, "Route selection for railway passengers: a multi-objective model and optimization algorithm," Journal of Transportation Systems Engineering and Information Technology, vol. 13, no. 5, pp. 72-100, 2013.

[9] J. Wu, Y. Qu, H. Sun, H. Yin, X. Yan, and J. Zhao, "Datadriven model for passenger route choice in urban metro network," Physica A: Statistical Mechanics and Its Applications, vol. 524, pp. 787-798, 2019.

[10] Y. Zhang, E. Yao, J. Zhang, and K. Zheng, "Estimating metro passengers' path choices by combining self-reported revealed preference and smart card data," Transportation Research Part C: Emerging Technologies, vol. 92, pp. 76-89, 2018.

[11] X. Xu, L. Xie, H. Li, and L. Qin, "Learning the route choice behavior of subway passengers from AFC data," Expert Systems with Applications, vol. 95, pp. 324-332, 2018.

[12] D. Earnhart, "Do travel cost models value transportation properly?" Transportation Research Part D: Transport and Environment, vol. 8, no. 6, pp. 397-414, 2003.

[13] B. De Borger and M. Fosgerau, "The trade-off between money and travel time: a test of the theory of reference-dependent preferences," Journal of Urban Economics, vol. 64, no. 1, pp. 101-115, 2008.

[14] A. C. Deserpa, "Microeconomic theory and the valuation of travel time: some clarification," Regional and Urban Economics, vol. 2, no. 4, pp. 401-410, 1973.

[15] S. Hess, M. Bierlaire, and J. W. Polak, "Estimation of value of travel-time savings using mixed logit models," Transportation Research Part A: Policy and Practice, vol. 39, no. 2-3, pp. 221-236, 2005.

[16] L. Fu, D. Sun, and L. R. Rilett, "Heuristic shortest path algorithms for transportation applications: state of the art," Computers \& Operations Research, vol. 33, no. 11, pp. 33243343, 2006.
[17] S. Raff, "Routing and scheduling of vehicles and crews: the state of the art," Computers \& Operations Research, vol. 10, no. 2, pp. 163-211, 1983.

[18] P. Modesti and A. Sciomachen, "A utility measure for finding multiobjective shortest paths in urban multimodal transportation networks," European Journal of Operational Research, vol. 111, no. 3, pp. 495-508, 1998.

[19] M. Samà, P. Pellegrini, A. D’Ariano, J. Rodriguez, and D. Pacciarelli, "Ant colony optimization for the real-time train routing selection problem," Transportation Research Part B: Methodological, vol. 85, pp. 89-108, 2016.

[20] F. Zong, Z. Jun, H. Zhang et al., "Calculation and application of value of travel time," Transportation System Engineering and Information, vol. 9, no. 3, pp. 114-119, 2009.

[21] R. B. Dial, "A probabilistic multipath traffic assignment model which obviates path enumeration," Transportation Research, vol. 5, no. 2, pp. 83-111, 1971. 


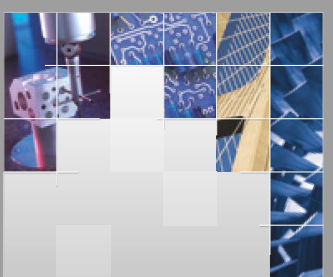

\section{Enfincering}
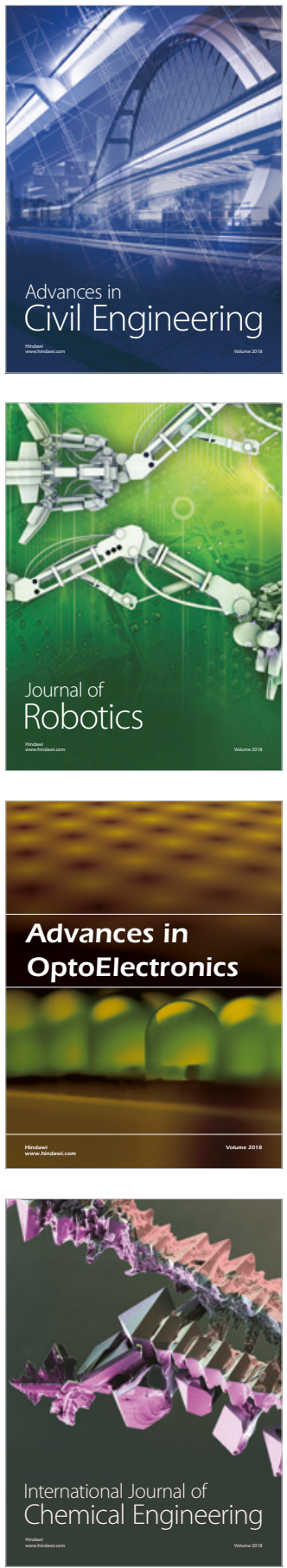

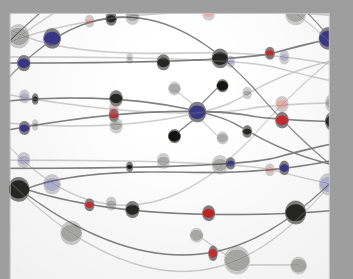

\section{Rotating \\ Machinery}

The Scientific World Journal

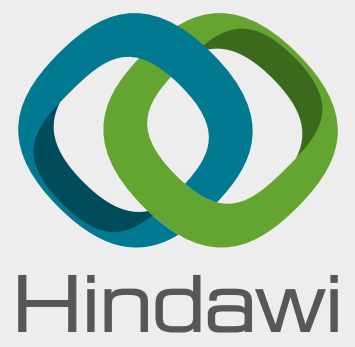

Submit your manuscripts at

www.hindawi.com
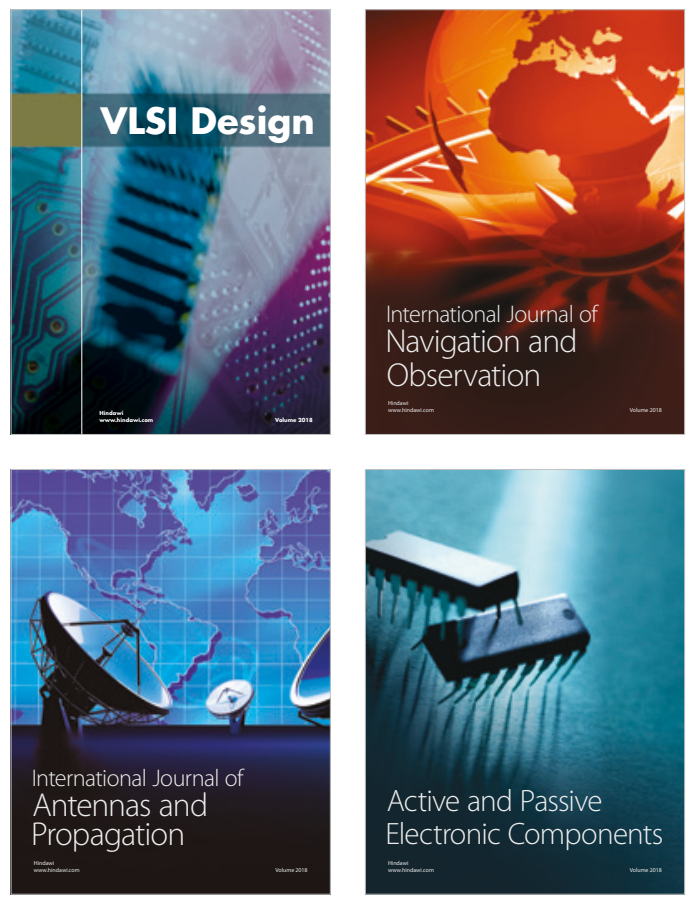
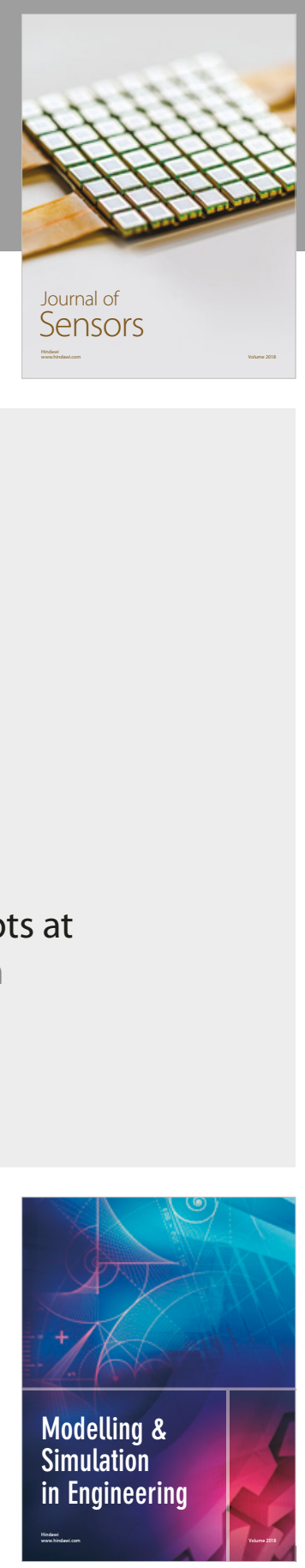

\section{Advances \\ Multimedia}
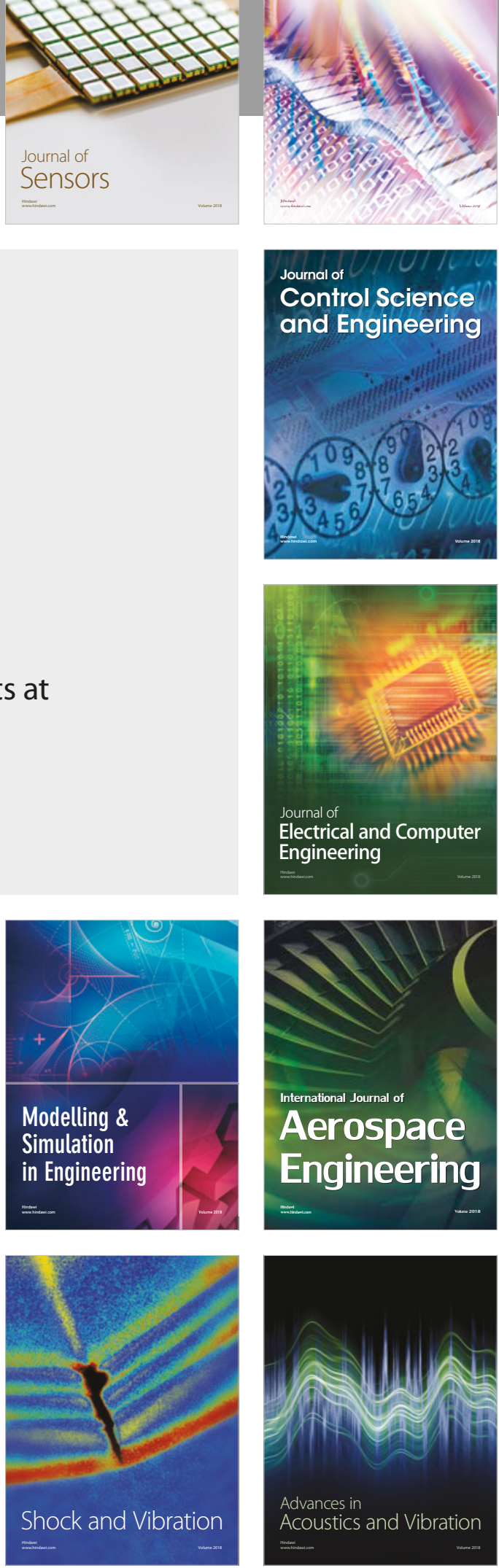